\title{
L'enseignement des réalités coloniales dans le roman de jeunesse Rêves amers
}

https://doi.org/10.29173/af29430

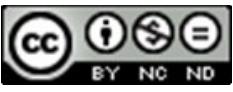

\section{Christophe Premat \\ https://orcid.org/0000-0001-6107-735X \\ christophe.premat@su.se}

Université de Stockholm, Suède

Résumé. Le roman de jeunesse Rêves amers de Maryse Condé est paru pour la première fois dans le magazine Je bouquine en 1987 avant d'être repris par les éditions Bayard jeunesse en 2001. Portant sur l'expérience tragique de la migration, il s'attache à mettre en évidence la pérennité de l'esclavage social (Mbembe 2013). "Le roman francophone des Antilles apporte aussi un changement considérable dans la nature des êtres humains. Les hommes et les femmes qui en sont les héros n'appartiennent pas à une catégorie bien définie. Ils mettent à mal le concept de race. Ils sont le résultat d'influences diverses. Ils portent en eux des sangs multiples et sont souvent des métis, sensibles à la couleur de leur peau qui conditionne la qualité de leur existence » confiait récemment Maryse Condé dans un entretien mené par Roger Célestin (153). Cet ouvrage, qui était dans sa forme initiale paru peu de temps avant Traversée de la mangrove, traite d'Haïti et de la Caraïbe comme des espaces de révolte par rapport à la malédiction historique des rapports brutaux de la colonisation (Carruggi date?). Si le roman Rêves amers a eu une certaine réception dans le cadre de la littérature jeunesse et de la pédagogie, il reste relativement négligé des études littéraires universitaires. Pourtant, la référence à Haïti est centrale avec l'avènement de la première République noire indépendante du Nouveau Monde. Notre hypothèse est que Maryse Condé a proposé un ouvrage didactique destiné à former les jeunes générations pour qu'elles réinterrogent ce qui est enseigné dans une optique postcoloniale. Le contenu, les thèmes et le style de cet ouvrage lui ont servi de matrice pour la série de romans qui ont suivi. Notre étude portera sur l'analyse de la relation entre la mort et le rêve pour dégager un positionnement fondamental sur la manière de rendre compte de relations socio-historiques issues du colonialisme. Dans ce cadre, Haïti demeure la promesse d'une émancipation inachevée qui est enseignée aux jeunes générations. Ces œuvres semblent négligées par la critique peut-être parce que leur facture didactique est beaucoup plus nette. 
Mots clés : Haïti, République noire, Maryse Condé, aliénation coloniale, roman jeunesse, loas, imaginaire vaudou.

\begin{abstract}
Maryse Condé's youth novel Rêves amers appeared for the first time in the magazine Je bouquine in 1987 before being taken up by the Bayard youth editions in 2001. Dealing with the tragic experience of migration, it highlights the recurrent reality of social slavery (Mbembe 2013). "The French-language novel from the Antilles also brings about a considerable change in the nature of human beings. The men and women who are its heroes do not belong to a well-defined category. They undermine the concept of race. They are the result of various influences. They carry multiple bloods within them and are often half-breeds, sensitive to the color of their skin which conditions the quality of their existence " recently confided Maryse Condé in an interview conducted by Roger Célestin (153). This work, which was published in its initial form shortly before Crossing the mangrove, treats Haiti and the Caribbean as spaces of revolt against the historical curse of brutal reports of colonization (Carruggi). If the novel Reves amers has had a certain reception within the framework of children's literature and pedagogy, it remains relatively neglected in academic literary studies. However, the reference to Haiti is central with the advent of the first independent Black Republic in the New World. The hypothesis here is that Maryse Condé has proposed a didactic book intended to train the younger generations to question what is taught from a postcolonial perspective. The content, themes and style of this book served as the basis for the series of novels that followed. Our study will focus on the analysis of the relationship between death and dreaming to identify a fundamental position on how to account for socio-historical relationships resulting from colonialism. In this context, Haiti remains the promise of an unfinished emancipation which is taught to the younger generations. These works seem neglected by the critics perhaps because their didactic style is much clearer.
\end{abstract}

Keywords: Haiti, Black republic, Maryse Condé, colonial alienation, youth novel, loas, Vodoo imaginary.

\title{
INTRODUCTION
}

L Le 16 avril 2009, l'écrivaine Maryse Condé rencontrait des collégiens de l'établissement AndréDulin d'Aigrefeuille (Aquitaine, France) pour leur parler de deux romans de littérature jeunesse et adulte, Chiens fous dans la brousse et Rêves amers. Lors de cette rencontre, elle a demandé aux élèves de « faire l'effort de se rapprocher, par l'imagination, des enfants dont elle parle dans ses livres, qui travaillent, sont très pauvres et subissent les douleurs de la vie » (Sud-Ouest 22). Elle a répété ce message au fil de ses visites de classe comme pour ancrer le lien entre littérature et empathie. La littérature permet de rencontrer des personnages de milieux sociaux très différents, elle ouvre les horizons et crée des possibilités de comprendre des conditions sociales extrêmement précaires.

C'est dans cet esprit qu'avait été conçu l'ouvrage Rêves amers qui avait paru pour la première fois dans un magazine pour enfants entre 10 et 15 ans. Le magazine littéraire Je Bouquine édité par Bayard avait publié ce roman pour la première fois en mai 1987 et affichait en première page le titre « Un roman vrai, Haïti chérie de Maryse Condé ». Sur la couverture du magazine, le lecteur voit un paysage tropical des Caraïbes avec deux jeunes adolescentes haïtiennes et un garçon en arrière-plan. L'une des jeunes filles,

Alternative francophone

https://journals.library.ualberta.ca/af/index.php/af 
représentant certainement le personnage principal Rose-Aimée, se tient la tête en laissant couler deux larmes. Bruno Pilorget a pour sa part illustré la couverture du roman de Maryse Condé paru aux éditions Bayard Jeunesse en 2001 et réédité en 2005 et en 2009. Il a choisi d'esquisser une jeune fille haïtienne en souffrance semblant méditer sur un paysage de mer et de palmiers. Son corps courbé révèle les difficultés d'un destin maudit alors que ses mains croisées semblent attendre des jours meilleurs (voir Fig.1 : couverture).

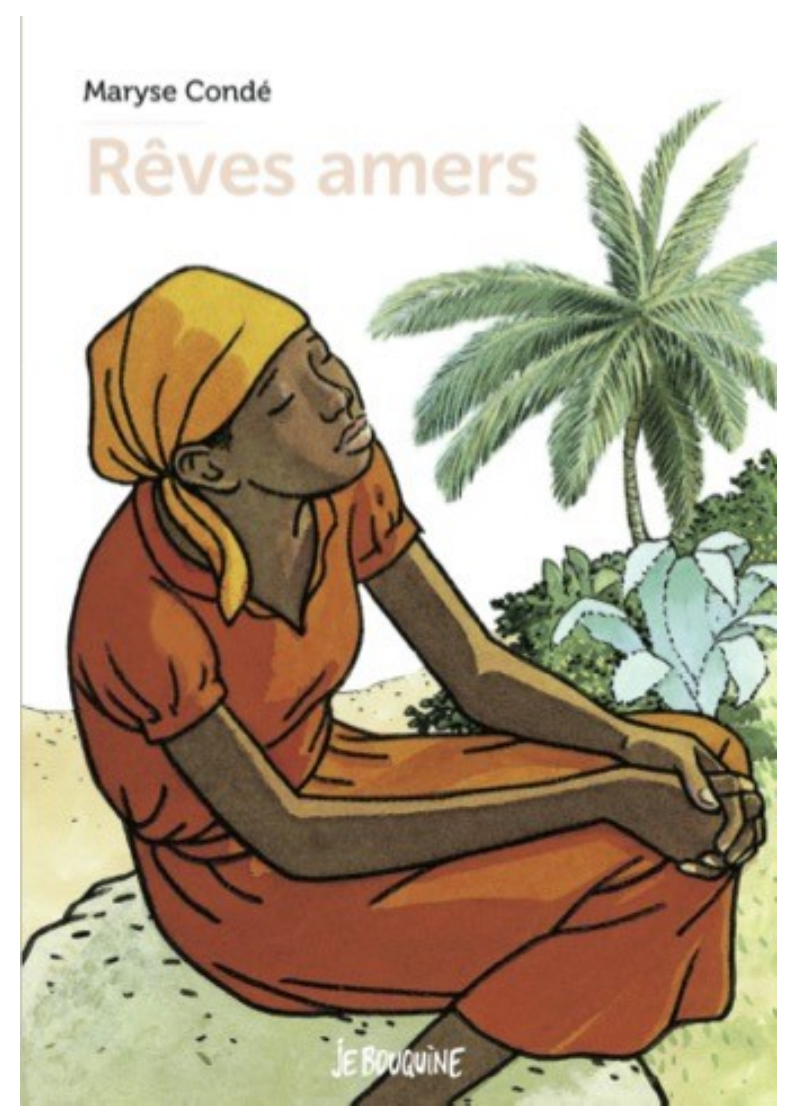

Figure 1 : page de couverture du livre Rêves amers. Source : « Rêves amers » de Maryse Condé, illustration de Bruno Pilorget, collection Je bouquine, CBayard Editions

Cet ouvrage, peu connu du grand public et négligé par les études universitaires, était paru avant l'un des grands chefs d'œuvre qui a fait la renommée de Maryse Condé, Traversée de la Mangrove. Haïti et la Caraïbe y sont traitées comme des espaces de révolte par rapport à la malédiction historique des rapports brutaux de la colonisation. Dans un entretien mené par Roger Célestin, Maryse Condé confiait récemment que :

Le roman francophone des Antilles apporte aussi un changement considérable dans la nature des êtres humains. Les hommes et les femmes qui en sont les héros n'appartiennent pas à une catégorie bien définie. Ils mettent à mal le concept de race. Ils sont le résultat d'influences diverses. Ils portent en eux des sangs multiples et sont souvent des métis, sensibles à la couleur de leur peau qui conditionne la qualité de leur existence. (153)

L'objectif de cet ouvrage, qui a surtout eu un public français hexagonal, est d'éveiller la conscience des jeunes publics à la multiplicité des récits et des manières de vivre. La référence à Haïti est centrale, car ce 
pays a été la première république noire au monde où les esclaves ont pu se libérer des colonisateurs après une longue révolution durant de 1791 à 1804. Notre hypothèse est que Maryse Condé a proposé un ouvrage didactique destiné à former les jeunes générations pour qu'elles réinterrogent dans une optique postcoloniale ce qui est enseigné. Comme le rappelle Élise Saincotille (58), Maryse Condé a écrit quatre romans dans le magazine Je bouquine, dont Haïti chérie, puis Victor et les barricades ${ }^{1}$, Chiens fous dans la brousse et Savannah Blues. Haïti chérie est un roman particulier dans le cadre des éditions Bayard qui vise à dépayser le jeune lecteur français pour l'installer dans un autre monde aux résonances magiques parce que lointaines. Les éditions Bayard ont pour ambition d'intéresser les jeunes publics à la lecture et organisent régulièrement des concours de nouvelles ${ }^{2}$. Ayant conscience du public visé, Maryse Condé a proposé un ouvrage introduisant un monde nouveau au lecteur français en l'invitant à s'intéresser à la dure réalité de l'esclavage et aux conséquences de la colonisation. Le contenu, les thèmes et le style de cet ouvrage lui ont servi de matrice pour la série de romans qui ont suivi. Notre étude portera sur l'analyse de la relation entre la mort et le rêve pour dégager un positionnement fondamental sur la manière de rendre compte des relations socio-historiques et d'exposer des problématiques postcoloniales. Dans ce cadre, Haïti demeure la promesse d'une émancipation inachevée qui est enseignée aux jeunes générations. Ces œuvres semblent négligées par la critique peut-être parce que leur facture didactique est beaucoup plus nette. L'introduction d'une perspective postcoloniale dans les œuvres de littérature jeunesse est également une méthode originale qui a peu retenu l'attention de la critique universitaire.

\section{LA PLACE D’HAITI DANS LA LITTÉRATURE JEUNESSE FRANCOPHONE}

Si le champ des études postcoloniales a littéralement explosé depuis plus d'une trentaine d'années, permettant ainsi l'éclosion de concepts importants pour l'analyse littéraire, la question de la didactisation de ces principes s'est posée notamment pour des publics n'étant pas forcément familiers avec la confrontation au passé colonial et à ses manifestations contemporaines (Aggarwal). Lorsqu'il s'agit de publics adolescents, cette didactisation doit impérativement passer par un accompagnement à l'exercice critique. Le défi est de pouvoir travailler sur des « structures de sentiment » (André 45), c'est-à-dire des situations types permettant d'activer une interrogation et parfois une indignation. La prise en compte du lectorat est également un élément déterminant, car l'objectif est de toucher un public parfois non conscient de ses privilèges afin qu'il questionne les conséquences du système colonial qui reste sans doute l'un des plus grands scandales de l'histoire. Lilian Thuram, que l'on pourrait qualifier de militant antiraciste, a pour sa part créé une fondation d'éducation contre le racisme intégrant des perspectives différentes sur l'histoire afin que les enfants et les adolescents remettent en question les préjugés raciaux et coloniaux. Il a ainsi écrit le livre Mes étoiles noires (2011) qui permet de présenter l'histoire du monde à partir des personnalités noires marquantes. La publication en 2014 de sa bande dessinée autobiographique portant sur sa trajectoire lui a permis de mettre en lumière le phénomène du racisme et la mentalité coloniale (Thuram 2014). L'un des arguments principaux de Lilian Thuram est d'affirmer que le racisme est l'affaire de tout le monde et surtout de celles et ceux qui ne le subissent pas. En effet, le racisme s'exerce principalement contre les populations de couleur différente du blanc. Les Blancs ne souffrent pas du racisme et n'imaginent pas les difficultés dans lesquelles certaines personnes se trouvent

${ }^{1}$ «Victor et les barricades », Je bouquine, nº61, mars 1989.

2 « Prix littéraire pour les collégiens », La Nouvelle République, 27 juin 2014, p. 37.

Alternative francophone

https://journals.library.ualberta.ca/af/index.php/af 
face à ce problème. Le fait d'avoir ce privilège doit être réinterrogé pour que les personnes ne le subissant pas puissent à leur tour le dénoncer et éprouver de l'empathie vis-à-vis des victimes du racisme.

Il semble que Maryse Condé ait investi le terrain de la littérature jeunesse pour transmettre ce sentiment d'indignation devant l'injustice des destins. Haïti est l'exemple historique important de la première république noire ayant un désir profond d'émancipation vis-à-vis du système esclavagiste. Maryse Condé rappelle l'importance de cet exemple historique dans Rêves amers : «Même si elle ne l'avait jamais visitée, Rose-Aimée savait que cette ville [Gonaïves] était le symbole de l'indépendance. C'est là que l'esclave révolté devenu général, Dessalines, avait déchiré le drapeau français et jeté à la mer la partie blanche, créant ainsi le drapeau d'Haïti rouge et bleu » (18).

Cette histoire est également racontée par Lilian Thuram qui décrit l'action de Dessalines de la manière suivante : «le 1er janvier 1804, Dessalines proclame l'indépendance et annonce que le nouvel État s'appellera Ayiti, le nom amérindien d'Haïti, restituant l'île à ses origines et rendant hommage à une population autochtone indienne quasi exterminée dès l'arrivée des Blancs » (Mes étoiles noires 107-108). En d'autres termes, les allusions historiques sont présentes dans le texte de Maryse Condé pour rappeler la façon dont le colonialisme a mis en berne toute volonté d'émancipation. Dans un autre passage, ce désir haïtien d'émancipation est symbolisé par les révoltes historiques des esclaves Makandal et Boukman (Rêves amers 60). La grande figure de Toussaint Louverture n'est cependant pas évoquée, mais Dessalines s'était joint à la révolte de Boukman en 1791 en suivant les traces de Toussaint Louverture (Mes étoiles noires 106; Ollivier 66).

Rose-Aimée revit de manière banale la condition de ces Pères fondateurs, comme si la construction de ce récit national d'émancipation ne pouvait se limiter aux faits et gestes de ces quelques grands hommes. Rose-Aimée est l'héroïne de cette histoire, elle est cette jeune fille qui prend conscience de la tragédie de la grande histoire ayant assujetti certains peuples (Meehan 295). En réalité, Rose-Aimée est l'incarnation de cette voix subalterne qui retrouve les racines de sa nation et réactualise d'une certaine manière ce désir historique d'émancipation (Glover 31). Elle apprend à faire face à Monsieur Modestin, patron au sein de la chaîne américaine «Kentucky Fried Chicken » (Rêves amers 51). Dans sa condition de domestique, la révolte de Rose-Aimée s'effectue avec la plus grande sérénité : «Comme Rose-Aimée, agenouillée sur le sol, s'apprêtait à attirer vers elle le seau d'eau mousseuse, d'un coup de pied monsieur Modestin l'envoya valser à l'autre bout de la pièce. Un grand calme s'empara de Rose-Aimée. Elle qui avait peur de tout brusquement se sentit forte. D'où lui venait cette détermination, ce courage de se lever et de faire face à monsieur Modestin? » (Rêves amers 59).

Rose-Aimée ne se résigne pas à subir l'humiliation, elle refuse avec dignité cette brutalisation (Mbembe 31). Ainsi, en choisissant une héroïne noire des Caraïbes, Maryse Condé fait sentir au jeune lecteur le poids de ces injustices historiques. Aimé Césaire avait en 1939 dans son Cahier d'un retour au pays natal mis en évidence la manière dont Haïti était le symbole de la négritude.

Et mon île non-clôture, sa claire audace debout à l'arrière de cette polynésie, devant elle, la Guadeloupe fendue en deux de sa raie dorsale et de même misère que nous, Haïti où la négritude se mit debout pour la première fois et dit qu'elle croyait à son humanité et la petite queue de la Floride où d'un nègre s'achève la strangulation, et l'Afrique gigantesquement chenillant jusqu'au pied hispanique de l'Europe, sa nudité où la mort fauche à larges andains. (23) 
$\mathrm{Au}$ fond, le destin de Rose-Aimée épouse cette malédiction à la fois géographique et historique où la couleur inscrit le stigmate de la trajectoire sociale ${ }^{3}$. La malédiction rappelle en filigrane la tragédie des destinées humaines, notamment celles des populations qui ont du mal à transcender leur condition sociale initiale, d'autant plus que la couleur de peau détermine la qualité de leur existence (Premat 4).

Le terme de « postcolonial » peut parfois prêter à confusion dans notre propos, car il ne s'agit pas de s'intéresser à une période historique succédant aux colonisations officielles, mais d'analyser les structures narratives reflétant l'aliénation de certaines sociétés (Moura 41). Rêves amers est un livre relativement méconnu qui est publié dans un contexte où la littérature postcoloniale francophone connaît une forte croissance. Le défi est de toucher un public jeune pour lui montrer les ravages du colonialisme sans tomber dans une quelconque forme d'exotisme colonial (Ménil 23). Si le colonialisme désigne de manière générique la domination d'un pays par un autre gouvernement, ses formes historiques et sociales sont très diverses (Loomba 35). Dans le cas de la présentation de ces formes sociales brutales dans la littérature jeunesse, il importe de partir d'utiliser Haïti comme le symbole fort de la révolte d'esclaves et de la première république noire dans le monde.

D'autres œuvres plus récentes de littérature jeunesse ont choisi de présenter la dure réalité d'Haïti sous le règne des tontons macoutes, les milices à la solde du dictateur Duvalier (Agnant 2003; Temple). Haïti est très présente dans les contes locaux en créole et en français comme le témoignent certaines publications (Contes dramatiques 1; Contes dramatiques 2; Tessonneau), même si Rêves amers demeure l'une des premières histoires publiées pour un public francophone en dehors d'Haïti. Par la suite, Mimi Barthélémy a également mis en scène les contes d'Haïti avec notamment le personnage de Bouki qui est évoqué dans Rêves amers (Barthélémy 2008; Barthélémy et al. 2011). D’autres grands écrivains haïtiens comme Marie-Célie Agnant ${ }^{4}$ et Dany Laferrière ${ }^{5}$ ont également publié des ouvrages pour la jeunesse.

Les maisons d'éditions québécoises La bagnole et Les 400 coups publient également des œuvres de littérature jeunesse autour d'Haïti en introduisant des illustrations et parfois des paroles d'enfant comme ce fut le cas avec la publication de Haïti, mon pays: poèmes d'écoliers haïtiens (Rogé). Cette série d'ouvrages est relativement récente, Maryse Condé inaugurant avec Rêves amers un double geste consistant d'une part à introduire Haïti dans la littérature jeunesse et d'autre part à remettre en question les catégories psychologiques et politiques du colonialisme (Bonnet 7). Véronique Bonnet rapporte la manière dont Maryse Condé entrevoit son roman Rêves amers dans l'un de ses entretiens avec Françoise Pfaff :

J'ai écrit pour la jeunesse pour une raison simple : les enfants me paraissent terriblement intelligents, mais il faut savoir attirer leur attention sur un fait ou sur un autre [...]. Je me suis donc dit que parler aux enfants du monde tel qu'il est, atroce, dur à vivre, et leur parler de la vie compliquée et scélérate, est une gageure que j'entreprends de bon gré pour les faire réagir. Par exemple, comment parler aux enfants de l'esclavage ! Écrire pour la jeunesse est un désir de lui apporter une réflexion sur le monde tel qu'il a été ou tel qu'il est. (Pfaff 157-158 cité dans Bonnet 2)

\footnotetext{
${ }^{3}$ Victor Hugo avait rendu hommage à cette république noire d'Haïti dans Actes et Paroles: « Haïti, dès 1792, en affranchissant les noirs, a fait triompher ce principe qu'un homme n'a pas le droit de posséder un autre homme » (637).

${ }^{4}$ Alexis d'Haïti (1999); Alexis, fils de Raphaël (2000); L'Oranger magique : Conte d'Haïti (2003).

${ }^{5}$ Je suis fou de Vava (2013); Le baiser mauve de Vava (2014).
} 
Ici, Maryse Condé prend conscience de la difficulté de la littérature jeunesse qui pourrait cacher le monde tel qu'il est aux enfants. Raconter des histoires, utiliser le conte pourraient être alors des détours pour éviter le scandale historique majeur que constitue le colonialisme; or, Maryse Condé n'a jamais cédé à ce piège et a constitué une œuvre de littérature jeunesse qui affronte les questions de domination raciale et sociale, que ce soit dans Rêves amers ou bien dans Hugo le terrible (1991), Chiens fous dans la brousse (2008), À la courbe du Joliba (2006) et Savannah blues (2009a).

\section{LA CONSTRUCTION DU RÉCIT}

Le récit est construit autour du personnage principal, une jeune haïtienne, Rose-Aimée. Son destin est contraint, il est de devenir la domestique de riches propriétaires terriens. Le récit est ainsi construit en deux séquences principales avec d'abord le départ du village de Limbé vers Port-au-Prince puis l'impossible voyage vers l'Amérique. Plus les conditions de vie s'avèrent difficiles, plus Rose-Aimée va s'enfermer dans une aliénation de plus en plus forte avec le rêve d'une vie hors de portée. Comme l'écrivait Frantz Fanon, " nous comprenons [...] pourquoi le Noir ne peut se complaire dans son insularité. Pour lui il n'existe qu'une porte de sortie et elle donne sur le monde blanc » (41). Cette expérience tragique de l'esclavage, le rêve de la sécurité matérielle s'inversant dans une condition domestique indépassable, résonne comme une forme de malédiction pour Rose-Aimée. Le lecteur pressent rapidement que le destin de Rose-Aimée ne lui appartient pas et que l'expérience migratoire est condition même de la survie.

Le livre est construit autour de cinq chapitres, «Le départ de Rose-Aimée », " Chez madame Zéphyr », « Les sans-abri », «Un patron terrifiant» et « Un bateau dans la nuit ». Chaque chapitre se termine par une réflexion sur la nouvelle étape à franchir dans la vie de Rose-Aimée, l'histoire se terminant par la mort. Ainsi, le premier chapitre s'achève sur la nouvelle destination de Rose-Aimée : «Timidement, Rose-Aimée tira de son corsage le chiffon de papier qui portait l'adresse de Madame Zéphyr et interrogea un passant. - Laisse voir! Mais c'est à Pétionville que tu dois aller! Là-haut dans la montagne » (Rêves amers 24).

L'adverbe « timidement » qui inaugure la phrase montre à quel point Rose-Aimée ne maîtrise pas son destin, comme trimbalée d'une adresse à l'autre. Le second chapitre s'achève sur les pensées de RoseAimée : «Pendant tout le trajet, une résolution s'était fortifiée au cœur de Rose-Aimée. Elle ne retournerait pas chez madame Zéphyr les mains vides. Celle-ci n'était-elle pas capable de la faire arrêter par la police? Après tout, elle savait repasser, cuisiner, faire le ménage, elle saurait bien trouver une autre place où elle serait mieux traitée... » (Rêves amers 37).

Le discours indirect libre caractérise cette volonté d'avoir une prise sur le destin pour éviter les mauvaises rencontres et les exploitations en tout genre. En l'occurrence, le livre est tendu vers cette tentative de résolution : comment Rose-Aimée peut-elle échapper à sa condition soumise?

La fin du chapitre trois porte sur la ville de Port-au-Prince que Rose-Aimée a apprivoisée avec moins de crainte : «Et ce n'était pas seulement les quartiers résidentiels que Rose-Aimée chérissait, mais aussi les bidonvilles, ceinture puante où les enfants piétinaient dans la gadoue à côté des chiens maléfiques et des adultes, que la misère vieillissait vite. Une ville, c'est comme un être humain. Elle possède sa personnalité, son caractère, et on s'y attache! » (Rêves amers 50). 
Dans ce passage, outre les métaphores marquantes comme « ceinture puante » pour décrire les périphéries délaissées, l'expression « la misère vieillissait vite » est moins courante avec l'usage plus rare du verbe « vieillir » comme verbe transitif où la misère est le sujet et les adultes les objets. Le discours indirect libre mêle en filigrane la voix de l'auteure décrivant de manière recherchée la ville de Port-au-Prince et les pensées de Rose-Aimée s'exprimant comme une adolescente (« on s'y attache! »). La phrase « Une ville, c'est comme un être humain » acquiert une forme de vérité générale équivalant à un précepte. Le chapitre suivant s'achève également sur une forme proverbiale ayant cette fois davantage une valeur de maxime.

Pourtant, le spectacle de toute cette misère ne découragea pas Rose-Aimée. Au contraire. Elle sentait naître en elle une volonté toute neuve. La vie, c'est comme une bête qu'il faut dompter. Il faut bander ses muscles comme un pêcheur mettant à l'eau une pirogue rétive. À tout moment, la lame risque de la submerger, de l'emporter. Néanmoins, il tient bon. Lisa avait raison. Il fallait partir pour Miami où, à n'en pas douter, la vie aurait un autre goût. (Rêves amers 62)

Le chapitre 4 s'achève sur le même refrain de la misère avec une maxime élaborée au fil de la migration de Rose-Aimée. Les phrases sont extrêmement courtes, les formes elliptiques permettant d'insister sur la résilience dont Rose-Aimée fait preuve. En effet, la succession de ces phrases courtes montre à quel point Rose-Aimée est de moins en moins passive, déterminée à œuvrer pour sa liberté. Il n'y a pas de place pour ses états d'âme, étant donné que le récit privilégie l'action.

Le livre s'achève sur l'échec du voyage et la mort devenue la métaphore d'une libération absolue, comme si la mer remettait les compteurs à zéro sans faire de différence.

Et la mer roula ces déshérités dans son suaire. Elle para leur corps d'algues, ouvragées comme des fleurs, suspendit à leurs oreilles des boucles d'oreilles de varech. Elle chanta de sa voix suave pour calmer les terreurs des enfants, de Rose-Aimée et de Lisa, et, les yeux fermés, ils glissèrent tous dans l'autre monde. Car la mort n'est pas une fin. Elle ouvre un au-delà où il n'est ni pauvres ni riches, ni ignorants ni instruits, ni Noirs, ni mulâtres, ni Blancs... (Rêves amers 80)

Cette image tragique présente la mort de manière positive, la personnification de la mer insistant sur le dernier instant des enfants au cours du naufrage. La présentation met en évidence une forme de dernier sacrement comme si les enfants étaient habillés pour accueillir le seul destin possible. La mer semble refléter les parures que pouvaient porter les gens sur la terre d'Haïti. Cette description n'est pas sans rappeler celle de l'écrivain haïtien Émile Ollivier évoquant pour sa part la terre haïtienne. « La terre d'Haïti me portait, me transportait; elle me costumait de mille broderies, me couronnait de mille pierreries somptueusement. Et j'allais à grands pas, avide et peu soucieux de l'orage qui nous attendrait au tournant » (102). La mort est tragique, mais en même temps, elle transfigure ce destin haïtien en lui donnant une signification doublement positive : la mort rend la dignité à Rose-Aimée en la parant de toutes les couleurs d'Haïti et elle indique en outre le sacrifice des générations passées en vue d'une promesse d'émancipation portée depuis les débuts de la première république noire. Néanmoins, cette promesse est constamment trahie et nous pouvons voir dans cette description une ironie amère comme si la mort présentait le négatif photographique d'un destin impossible dans le monde des vivants.

Alternative francophone

https://journals.library.ualberta.ca/af/index.php/af 


\section{LE RÊVE IMPOSSIBLE}

Le titre Rêves amers est polysémique, l'adjectif « amers » renvoyant à la frustration d'un destin jamais atteint, mais également à la mer comme si le rêve n'avait pas de destination fixe. Rose-Aimée prend conscience que les rêves ne sont jamais réalisés, elle intériorise par le truchement du discours indirect le rêve de son frère Romain qui était parti pour survivre et qui n'avait probablement pas survécu. « Son rêve était de revenir bien vite afin de bâtir une petite maison avec des tôles sur le toit. Seulement voilà, il n'était jamais revenu...» (Rêves amers 12). Ce frère disparu traverse l'esprit de Rose-Aimée quand elle est domestique chez Madame Zéphyr : «Cela rappela à Rose-Aimée son frère Romain, disparu quelques années plus tôt dans l'enfer de la canne à sucre! Elle regarda, le cœur serré, tous ces miséreux obligés de quitter leur pays natal pour connaître la dure loi de l'étranger » (Rêves amers 31). Cette maison avec des « tôles sur le toit » que Rose-Aimée présente rapidement pour évoquer le rêve de son frère, renvoie à la dimension de la sécurité matérielle, de la survie.

L'exploitation des cannes à sucre impose la réalité sociale de l'esclavage dans ce roman. Le frère de Rose-Aimée a travaillé dans une plantation en vue d'une libération illusoire. Son modeste rêve ne s'est jamais réalisé. Fanon analysait les rêves de jeunes Antillais pour montrer l'intériorisation de ce complexe d'infériorité. Dans un passage où il évoque l'approche de Jung en termes de transitivité psychanalytique, voici ce qu'il écrit : « dans l'inconscient collectif de l'homo occidentalis, le nègre, ou, si l'on préfère, la couleur noire, symbolise le mal, le péché, la misère, la mort, la guerre, la famine » (154). Non seulement le rêve, c'est-à-dire le désir d'avenir du frère de Rose-Aimée est limité à un petit espace de sécurité matérielle et de survie, mais son destin tragique reflète la condition haïtienne et plus largement la condition caribéenne. «L'enfer de la canne à sucre » est la métaphore fondamentale de l'esclavage, celle qui imprègne la totalité de la société haïtienne : «Le Conseil d'État du sucre sollicite du gouvernement haïtien pour la récolte sucrière 1985-1986, par lettre adressée à l'ambassade d'Haïti à Santo Domingo, l'embauche de quinze mille ouvriers agricoles pour les besoins des usines sucrières de l'État dominicain... » (Rêves amers 31).

Dans l'ouvrage de Maryse Condé, le lecteur découvre des personnages migrant pour des raisons essentiellement économiques, à savoir pour assurer la survie de leur famille ${ }^{6}$. Le discours indirect libre est souvent utilisé pour exprimer certaines questions que se pose Rose-Aimée sur l'état des relations sociales à Haïti. Ces questions ne trouvent pas de réponse adéquate : «Pourquoi des peuples sont-ils riches, et d'autres si pauvres qu'ils doivent aller chercher hors de leur pays natal des moyens de subsister? RoseAimée eut beau tourner cette question dans sa tête, elle ne lui trouva pas de réponse » (Rêves amers 42).

Cette interrogation posée dans des termes simples d'une jeune adolescente est en réalité centrale dans le texte. La révolution haïtienne de 1986 qui a chassé le dictateur Jean-Claude Duvalier est évoquée dans le livre avec notamment la mention de Gonaïves qui fut le lieu où les manifestations anti-Duvalier ont émergé (Rêves amers 18).

\section{LA PRÉSENCE DE L’ALIÉNATION COLONIALE}

La littérature jeunesse a peut-être un atout dans la possibilité d'exploiter la fibre du conte avec les personnages présentés sous forme d'adjuvants et d'opposants. En fait, ce genre narratif est même assez adapté pour pouvoir rendre compte de l'aliénation coloniale et de ses effets sur la psychologie

\footnotetext{
${ }^{6}$ La migration économique des ouvriers agricoles haïtiens est souvent rapportée par la presse. Jean-Michel Caroit, «République Dominicaine : expulsés ou partis volontairement, vingt-cinq mille Haïtiens ont dû regagner leur pays », Le Monde, 19 août 1991.
} 
individuelle et collective. Dans le système social hérité du colonialisme, le « nègre » est celui qui est fondamentalement rejeté. L'incipit du chapitre 2 chez madame Zéphyr est similaire à celui du chapitre 5 de Frantz Fanon dans Peau noire, masques blancs. Le tableau 1 permet de comparer les deux incipit pour montrer l'expérience première de cette anomalie.

Tableau 1: Comparaison de deux incipit chez Maryse Condé

\begin{tabular}{|l|l|}
\hline $\begin{array}{l}\text { Chapitre } 2 \text { de Rêves amers, «Chez madame } \\
\text { Zéphyr » }\end{array}$ & Chapitre 5 de Peau noire, masques blancs \\
\hline «- Regardez-moi cette petite négresse, & " "Sale nègre !" ou simplement : “Tiens, un \\
cette petite paresseuse qui dort! Est-ce que tu & nègre !". J'arrivais dans le monde, soucieux \\
crois que je t'ai prise à mon service pour & de faire lever un sens aux choses, mon âme \\
dormir? La gifle de madame Zéphyr envoya & pleine du désir d'être à l'origine du monde, \\
Rose-Aimée rouler à l'autre bout du lit. » & et voici que je me découvrais objet au milieu \\
(Rêves amers 25) & d'autres objets » (Fanon 88). \\
\hline
\end{tabular}

Dans les deux incipit, l'image inversée du système colonial avec la dévalorisation a priori du nègre apparaît comme un choc, la « gifle » de Madame Zéphyr, au-delà de la maltraitance physique et psychique, venant installer cette représentation figée des catégories coloniales. Fanon fait exactement le constat de ce regard posé sur le nègre, comme si ce regard niait la possibilité de reconnaître le « nègre » comme un autre : «Enfermé dans cette objectivité écrasante, j’implorai autrui. Son regard libérateur, glissant sur mon corps devenu soudain nul d'aspérités, me rend une légèreté que je croyais perdue et, m'absentant du monde, me rend au monde » (Fanon 88).

Dans le cas de Rêves amers, le racisme a plusieurs couches aux Antilles entre les Blancs, les mulâtres et les nègres selon le degré d'éloignement vis-à-vis de la condition d'esclave. Madame Zéphyr est une « mulâtresse » décrite de la manière suivante : « une mulâtresse à la peau couleur de miel, aux longs cheveux soyeux qu'elle peignait longuement comme la déesse de l'Artibonite, avant de les rouler en un lourd chignon sur sa nuque » (Rêves amers 26) ${ }^{7}$.

Cette hiérarchie des conditions raciales renvoie à la réalité des Caraïbes avec la présence d'une bourgeoisie mulâtre : « il y avait aussi aux Antilles ce petit hiatus qui existe entre la békaille, la mulâtraille et la négraille » (Fanon 89). Les Blancs sont présents en bout de chaîne avec un Blanc qui accompagne la tentative de migration de Rose-Aimée vers la Floride (Rêves amers 71). Le patron du « Kentucky Fried Chicken » est également un « mulâtre en complet-veston » (Rêves amers 52). Le mulâtre se distingue du nègre, car il faisait partie des métis qui peuvent être affranchis de l'esclavage s'ils ont un père blanc. La rencontre avec le nègre est déjà caractérisée par une forme d'humiliation moins forte que celle résultant du contact avec les Blancs (Zobel 290).

Maryse Condé était pleinement consciente de ces catégories d'autant plus qu'elle avait soutenu une thèse en 1976, sous la direction de René Étiembre, sur le stéréotype du noir dans la littérature antillaise.

${ }^{7}$ L'Artibonite qui représente un grand fleuve de l'Ouest d'Haïti, est également le sujet de nombreux contes à Haïti (Wainwright 44).

Alternative francophone

https://journals.library.ualberta.ca/af/index.php/af 
Le peuple antillais est peut-être le seul qui n'ait pas choisi le lieu de sa résidence, mais à qui il a été imposé. Car, il ne s'est pas rendu dans les diverses îles des Caraïbes, sous la conduite de chefs, à la recherche de conditions de vie meilleures, de terres plus fertiles, de gibier plus abondant. Il y a été emmené de force à bord des Négriers. Parvenu à destination, il n'a pas eu la liberté d'organiser son univers. Il a été soumis à la canne à sucre, elle-même assez ironiquement, objet d'importation, qui a délimité l'espace laissé aux cultures vivrières au sein d'un système de production bien défini. (Stéréotypes du noir 2)

Rose-Aimée ne trouve jamais la liberté, car son destin est déjà écrit, elle n'arrive pas à le déjouer. En d'autres termes, la commodité de la littérature jeunesse tient au fait qu'elle puisse devenir une littérature à thèse. Tout ce que Maryse Condé critique dans sa thèse, sur les perceptions et les préjugés des ethnologues européens sur les Antilles, se retrouve présenté de manière didactique dans ce roman.

\section{L'IMAGINAIRE VAUDOU DANS LE RÉCIT}

L'une des résistances à cette empreinte de l'imaginaire colonial est le recours aux loas, ces esprits intermédiaires entre les dieux et les hommes, adoucissant les effets de l'aliénation coloniale (Rêves amers 15). L'introduction de cet imaginaire sacré caraïbéen est importante pour le jeune lecteur, le loa pouvant annoncer l'avenir. Le loa symbolise partiellement cet adjuvant protecteur que l'on implore pour avoir de meilleures conditions de vie. Lorsque celui-ci est indifférent et ne répond pas, alors l'individu est livré seul à son destin : «Peut-être un loa, le bon papa Legba ou mère Ersulie que l'on dit si belle avec ses robes colorées et ses colliers de fleurs, aurait-il daigné lui parler, lui révéler ce que serait sa vie à Port-auPrince auprès de cette famille Zéphyr? » (Rêves amers 16).

Le loa est le compagnon imaginaire que l'on peut invoquer et implorer en vue d'un futur meilleur, mais ce rituel n'aboutit jamais à une transformation du destin. Papa Legba est un vaudou haïtien présent dans la culture populaire. Jacques Roumain, l'un des grands écrivains haïtiens, a fait référence à Papa Legba dans son livre Les gouverneurs de la rosée de 1944 : «Va trouver Délira. Le reste, je ne l'ai pas entendu, les coqs chantaient, je me suis réveillée. C'était peut-être Papa Legba » $(38 ; 59 ; 79)$. Papa Legba est en fait cette divinité qui rappelle l'histoire tragique de l'esclavage : " Papa Legba est bien cet Iwa ou Loa, nommé Elegba à Cuba ou Eshu au Brésil. Doté de forces contradictoires, il est surtout connu pour avoir traversé l'Atlantique avec les esclaves et se placer aux intersections des avenirs. C'est l'incontournable intermédiaire entre l'ici et l'au-delà, qui symboliquement nommerait les terres perdues du Bénin, du Togo ou du Dahomey » (Delmeule 88).

Papa Legba contient le passé du traumatisme colonial marqué par l'esclavage, il est l'intercesseur entre les deux mondes pour pouvoir accompagner les personnes dans leur trajectoire. Rose-Aimée se réfère aux innombrables sanctuaires construits pour appeler l'esprit de Papa Legba qui fait rimer migration et mémoire. La migration n'est pas synonyme de perte d'identité, elle est chargée d'une histoire garantie par Papa Legba.

L'évocation d'Ersulie (écrit le plus souvent Erzulie) est à relever, car cette loa a en fait plusieurs visages dans la tradition vaudoue. Elle peut être associée à Erzulie-Fréda, la divinité de l'amour et du luxe (les « colliers de fleurs » renvoient à cette image), à Erzulie-Dantor, la femme noire représentée dans l'iconographie catholique comme étant une mère protectrice et Erzulie-gé-rouge incarnant la rébellion et la vengeance (Dayan 6). Ces figures sont entremêlées, même s'il y a une seule allusion à Erzulie dans Rêves amers.

Cet imaginaire religieux n'est pas convoqué pour introduire un quelconque exotisme esthétique, il incarne la résistance à l'imaginaire colonial qui enferme les colonisés dans une malédiction et une histoire unique. 
Jean-Paul Sartre, dans sa préface des Damnés de la terre de Frantz Fanon, associait les loas à l'aliénation religieuse des colonisés :

Ce qui était autrefois le fait religieux dans sa simplicité, une certaine communication du fidèle avec le sacré, ils en font une arme contre le désespoir et l'humiliation : les zars, les loas, les Saints de la Sainterie descendent en eux, gouvernent leur violence et la gaspillent en transes jusqu'à l'épuisement. En même temps ces hauts personnages les protègent : cela veut dire que les colonisés se défendent de l'aliénation coloniale en renchérissant sur l'aliénation religieuse. (Sartre 49)

Nous ne sommes pas sûrs que les loas redoublent l'aliénation des colonisés au sens où Sartre l'entendait lorsqu'il interprétait le livre de Frantz Fanon. Il semblerait au contraire que ces loas fonctionnent comme des adjuvants accompagnant Rose-Aimée vers la mort perçue comme transition vers un autre monde. Cela ne signifie pas qu'il y ait une forme de rédemption dans la mort, mais la mort vient supprimer l'ensemble des préjugés sociaux et raciaux. C'est la mer qui fait fonction de figure matricielle à la fin du récit pour atténuer la tragédie du récit. «Et la mer roula ces déshérités dans son suaire » (Rêves amers 80). Cette phrase contient un zeugme sémantique rattachant deux éléments disparates, typique du style de Maryse Condé. In fine, Rose-Aimée incarne un destin collectif de jeunes filles maudites tentant de fuir la misère de l'île et périssant dans leur voyage. La mer vient soulager, elle efface les humiliations, mais elle ne vient pas faire oublier ce passé colonial. Elle conserve ces corps maudits traînés d'un lieu à l'autre et leur redonne une beauté tragique.

\section{CONCLUSION}

Rêves amers de Maryse Condé est incontestablement une des premières œuvres de littérature jeunesse à présenter la réalité et les conséquences du colonialisme et de l'esclavagisme. La fiction est donc utilisée pour faire pénétrer les jeunes lecteurs dans cet univers où ils se rendent comptent des ravages de l'esclavage qui contraint les individus à se soumettre au caprice de dirigeants. L'héroïne de ce récit, RoseAimée, n'arrive pas à inverser ce déterminisme, d'autant plus que sa couleur de peau la place au plus bas de l'échelle sociale; elle est associée à cette « race maudite » (Traversée 85). Le fait d'avoir choisi Haïti pour retracer cette condition caraïbéenne n'est pas anodin non plus, puisque Haïti a été l'un des pays où le rêve d'une république noire avait émergé sur les idéaux de la Révolution française avant que les révoltes ne fussent matées. L'échec de cette émancipation est rappelé dans le destin tragique de Rose-Aimée qui, au-delà de son histoire, répète la grande histoire de ce pays qui a défié les puissances coloniales. Le jeune lecteur francophone européen est ainsi confronté à des questions qu'il ignore, portant sur la racialisation (classement selon les critères nègre / mulâtre / blanc) et d'une certaine manière la brutalisation des rapports sociaux. Il existe une intertextualité implicite avec les textes de Frantz Fanon qui décrivait l'intériorisation de l'infériorité des personnes colonisées. La dénonciation du scandale du colonialisme est profonde en ce que ce système sépare les individus et assigne des privilèges à un petit nombre au détriment des autres. Maryse Condé a choisi d'investir le champ de la littérature jeunesse pour présenter la perception des Caraibes comme étant un espace non choisi par ses habitants, un espace subi abritant le traumatisme des populations africaines déportées (Stéréotypes du noir 4). Le roman de Maryse Condé permet de présenter cette réalité coloniale lourde à des jeunes publics. Il fait la jonction entre les travaux théoriques de Maryse Condé avec notamment sa thèse de 1976 et ses romans à partir de la fin des années 1980. 


\section{BIBLIOGRAPHIE}

Aggarwal, Kusum, éditrice. Les Francophonies postcoloniales : textes et contextes. Langers International Pvt Ltd, 2016.

Agnant, Marie-Célie. L'Oranger magique : Conte d'Haïti. Les 400 coups, 2003.

—. Alexis, fils de Raphaël. Hurtubise. 2000.

—. Alexis d'Haïti. Hurtubise. 1999.

André, Sylvie. Pour une lecture postcoloniale de la fiction réaliste (XIX $X^{e}-X X^{e}$ siècle). Honoré Champion, 2018.

Barthélémy, Mimi. L'histoire d'Haïti racontée aux enfants. Istwa peyi dayiti ak ti mimi. Mémoire d'encrier, 2008.

Barthélémy, Mimi, Keraval, Gwen. De bouche à oreille, contes d'Haïti. Milan, 2011.

Bonnet, Véronique. « La littérature de la Caraïbe pour la jeunesse : des histoires à part ou l'histoire à part entière ? » Amnis, vol, 16, http://journals.openedition.org/amnis/3147.

Bonniol, Jean-Luc. La couleur comme maléfice. Albin, 1992.

Carruggi, Noëlle, éditrice. Maryse Condé. Rébellion et transgressions. Karthala, 2010.

Célestin, Roger. « Entretien avec Maryse Condé: quelques acquis et manques de la littérature francophone des Antilles. » Contemporary French and Francophone Studies, vol, 22, no 2, 2018, pp. 152-155.

Césaire, Aimé. La Poésie. Seuil, 2006.

Condé, Maryse. Stéréotype du noir dans la littérature antillaise - Guadeloupe-Martinique. Thèse de littérature comparée. Sorbonne Nouvelle, 1976.

Condé, Maryse. Rêves amers. Bayard éditions, 2009.

- Savannah blues. Sépia, 2009.

—. Chiens fous dans la brousse. Paris, Bayard Poche, 2008.

—. À la courbe du Joliba. Paris, Grasset Jeunesse, 2006.

—. «Pan-Africanism, Feminism and Culture. » Imagining Home: Class, Culture and Nationalism in the African Diaspora, édité par Sidney J. Lemelle, Robin D. G. Kelley. Verso, 1994, pp. 55-65.

—. Hugo le terrible. Saint-Maur-des-Fossés, Sépia, 1991.

—. Traversée de la mangrove. Paris, Seuil, 1989.

Dayan, Joan. « Erzulie : A women's history of Haiti. » Research in African Literatures, vol. 25, no 2, 1994, pp. 5-31.

Delmeule, Jean-Christophe. «La Divine Chanson : les musiques funambules ou Papa Legba aux carrefours des continents. »'Etudes littéraires africaines, vol. 44, 2017, pp. 79-94.

Fanon, Frantz. Peau noire, masques blancs. Seuil, 1952.

Glover, Kaiama, L. Haiti Unbound, A Spiralist Challenge to the Postcolonial Canon. Liverpool University Press, 2010. 
Hugo, Victor. Actes et paroles : Pendant l'exil, 1852-1870. Lévy, 1875.

Hyppolite, Michelson Paul. Contes dramatiques haïtiens. Tome II., Imprimerie de l'État. 1956.

—. Contes dramatiques haïtiens. Tome I. Imprimerie de l'État. 1951.

Kruegger Enz, Molly. «The Haitian Flight for Freedom in Maryse Condé's Rêves amers and MarieCéline Agnant's Alexis d'Haïti. » Contemporary French and Francophone Studies, vol. 22, no 5, 2018, pp. 553-561, https://doi.org/10.1080/17409292.2018.1580470

Laferrière, Dany, Normandin, Frédéric. Je suis fou de Vava. La Bagnole, 2013.

—. Le baiser mauve de Vava. La Bagnole, 2014.

Laroque, Lydie. " Haïti dans la littérature générale et de jeunesse contemporaine : étude comparative des romans L'autre face de la mer et Rêves amers ", Loxias, no 30, 2010, http://revel.unice.fr/loxias/index.html?id=6383

Loomba, Ania. Colonialism/Postcolonialism. Routledge, Taylor \& Francis Group, 2015.

Manson, Michel. «Julie Gouraud et Saint-Domingue : du roman familial au roman pour enfants. » Strence, 2012, http://journals.openedition.org/strenae/517

Mbembe, Achille. Le brutalisme. La découverte, 2020.

Mbembe, Achille. Critique de la raison nègre. La découverte, 2013.

Meehan, Kevin. « Romance and Revolution: Reading Women's Narratives of Caribbean Decolonization. » Tulsa Studies in Women's Literature, vol. 25, no. 2, 2006, pp. 191-306.

Ménard, Nadève. Écrits d'Haïti. Perspectives sur la littérature haïtienne contemporaine (1986-2006). Karthala, 2011.

Ménil, René. Tracées : identité, négritude, esthétique aux Antilles. Laffont, 1981.

Moura, Jean.-Marc. « Postcolonialisme et comparatisme. SFLGC, Bibliothèque comparatiste » 2019, http://sflgc.org/bibliotheque/moura-jean-marc-postcolonialisme-et-comparatisme/

Ollivier, Émile. Mille eaux. Gallimard, 1999.

Premat, Christophe. « Entre aliénation et déception identitaire : étude de la Traversée de la Mangrove de Maryse Condé », Karib - Nordic Journal for Caribbean Studies, vol. 5, no 1-2, 2020, http://doi.org/10.16993/karib.37

Rogé. Haïti, mon pays : poèmes d'écoliers haïtiens. Montréal, les éditions de la Bagnole, 2010.

Roumain, Jacques. Les gouverneurs de la rosée. Port-au-Prince, Imprimerie de l’État, 1944.

Saincotille, Élise. « C comme Condé ». Abécédaire insolite des francophonies. Édité par Chaulet-Achour, Christiane, et Brigitte Riéra. Pessac, Presses Universitaires de Bordeaux, 2012, pp. 57-76, http://books.openedition.org/pub/3584

Sartre, Jean.-Paul. « Préface. » Frantz Fanon, Les damnés de la terre. Maspéro, 1961, pp. 18-34.

Sud-Ouest, « Maryse Condé au collège », Section Pays Rochefortais, 27 avril 2009, p. 22.

Alternative francophone

https://journals.library.ualberta.ca/af/index.php/af 
Taleb-Khyar, Mohamed, B. «An interview with Maryse Condé and Rita Dove.» Callaloo, vol. 14, no. 2, 1991, pp. 347-366.

Temple, Frances. Cette nuit, on embarque. Flammarion, 1999.

Tessonneau, Louise. Éditrice. Contes créoles d'Haïti (textes rassemblés et traduits par Louise Tessonneau). Agence de coopération culturelle et technique, Conseil international de la langue française, 1980.

Thuram, Lilian. Notre histoire. Vol. 1. Delcourt, 2014.

—. Mes étoiles noires. De Lucy à Barack Obama. Philippe Rey, 2010.

Viala, Fabienne. «Maryse Condé et l'île qui se répète, poétique tragique postcoloniale dans Traversée de la mangrove. » Francofonia, no 61, 2011, pp. 125-140.

Wainwright, Édith. Culture Haïtienne à travers des textes choisis, Manuel d'enseignement. Educa Vision, 2001.

Wilson, Elizabeth. A. «Translating Caribbean Landscape.», Palimpsestes, no. 12, « Traduire la littérature des Caraïbes », 2000, pp. 8-17, https://journals.openedition.org/palimpsestes/1630

Zobel, Joseph. La Rue Cases-Nègres. Froissart, 1950. 